\title{
Desafíos teóricos y metodológicos en torno al documento de archivo como objeto digital en la red
}

\author{
Antonela Isoglio* \\ Diego Germán Vigna*
}

Artículo recibido:

15 de septiembre de 2020

Artículo aceptado:

19 de noviembre de 2020

Artículo de revisión

\section{Resumen}

La noción de documento de archivo presenta un rol central en la teoría y la metodología archivísticas. La difusión de las tecnologías de la información digital en las entidades productoras de documentos y los archivos implicó una serie de cambios, entre los que se encuentra la consideración acerca de la naturaleza del objeto de estudio e intervención. Sin embargo, las transformaciones más radicales se comenzaron a observar en las prácticas de archivo de objetos digitales en internet. Este artículo de revisión tiene el objetivo de analizar las premisas teóricas y metodológicas del concepto de documento de archivo, y contrastarlas con las peculiaridades que fueron surgiendo en torno a las prácticas

\footnotetext{
Universidad Nacional de Córdoba y Consejo Nacional de Investigaciones Científicas y Técnicas, Centro de Investigaciones y Estudios sobre Cultura y Sociedad CIECS (CONICET-UNC) aisoglio@unc.edu.ar diegovigna@unc.edu.ar INVESTIGACIÓN BIBLIOTECOLÓGICA, vol.35, núm. 87, abril/junio, 2021, México, ISSN:
} 2448-8321 pp. 129-150 
archivísticas de objetos digitales en internet. El trabajo se estructura en tres partes. Mientras que la primera presenta los presupuestos teóricos y metodológicos de dicho concepto dentro del paradigma dominante, la segunda los pone en relación con las propiedades emergentes de la práctica de archivo de objetos digitales en internet. Finalmente, la tercera parte aporta una reflexión acerca de los desafíos teóricos y metodológicos que se abren en el contexto actual, proponiendo algunas líneas de indagación para nuevas aproximaciones.

Palabras clave: Archivología; Tecnología de la Información; Internet

Theorerical and methodological challenges around the record as a digital object on the web

Antonela Isoglio and Diego Germán Vigna

\begin{abstract}
The notion of record (or archival document) plays a central role in archival theory and methodology. The diffusion of digital information technologies in the entities that produce documents and archives implied a series of changes, among which is the consideration of the nature of the object of study and intervention. However, the most radical transformations began to be observed in the practices of archiving digital objects on the internet. This review article aims to analyze the theoretical and methodological premises of the concept of record, and contrast them with the peculiarities that were emerging around the archival practices of digital objects on the internet. The work is structured in three parts. While the first presents the theoretical and methodological assumptions of the said concept within the dominant paradigm, the second puts them in relation to the emerging properties of the practice of archiving digital objects on the internet. Finally, the third part provides a reflection on the theoretical and methodological challenges that open up in the current context, proposing some lines of inquiry for new approaches.
\end{abstract}

Keywords: Archive Science; Information Technology; Internet 


\section{INTRODUCCIÓN}

A finales del siglo XX, la difusión masiva de las tecnologías de procesa1 miento y comunicación digitales en las instituciones archivísticas trajo aparejado un conjunto de cambios en el tratamiento y las formas de pensar las unidades documentales. Esto implicó el desarrollo de nuevas operaciones sociotécnicas y la modificación de las existentes, lo cual transformó también las funciones de los profesionales a su cargo (Marín Agudelo, 2012). En la dimensión conceptual, apareció un nuevo término, documento electrónico, para identificar la naturaleza de un objeto de estudio discernible de los documentos de archivo convencionales.

Más aún, la expansión global de internet en casi todas las esferas de la vida social profundizó las transformaciones ocurridas hacia el interior del paradigma archivístico. En este sentido, la noción de documento de archivo se vio profundamente trastocada en las diversas prácticas de $\operatorname{archivo}^{1}$ que comenzaron a desarrollarse en el entorno web (Kallinikos, Aaltonen y Marton, 2010; Pogaçar, 2016). Dicho concepto ha sido central en el paradigma archivístico dominante, ya que, como señaló una de las principales referentes teóricas, "no puede hablarse de archivos sin documentos" (Heredia Herrera, 1991: 121). Estos últimos constituyen la materia prima de los archivos, dando lugar a su existencia y haciendo necesaria la labor profesional del archivero (Heredia Herrera, 1991) en su concepción tradicional.

Según dichas premisas, los documentos de archivo no sólo son portadores de información, sino que además dan testimonio del desarrollo de las actividades realizadas por una persona física o jurídica, de las que se obtienen como resultado. En este sentido, toda actividad humana forma parte de un potencial acto de archivo: los productos de los hechos (históricos, culturales, políticos, artísticos) merecen ser organizados y preservados a largo plazo como evidencia del presente y el pasado (Alberch i Fugueras y Cruz Mundet, 1999).

Precisamente, el proceso secuencial históricamente atribuido al tratamiento archivístico conforma el cimiento sobre el cual ha sido posible reconocer a los documentos de archivo como testimonios materiales de hechos o actos de la entidad productora en el ejercicio de sus funciones (Subdirección General de los Archivos Estatales, 1995). Según las definiciones disciplinares, la confianza se ha sostenido sobre la estabilidad de los documentos, con

1 Optamos por el uso de esta mención porque la consideramos como la mejor adecuación conceptual y metodológica ante la complejización y diversificación que ha sufrido, justamente, la pregunta sobre qué es un documento de archivo. Tomamos a las prácticas de archivo, o prácticas archivísticas, como referencias que conjugan el tratamiento documental y los procesos de archivo en medio de la convivencia de soportes. 
base en soportes analógicos, y el resguardo de su valor archivístico. Partiendo de entidades fijas, la práctica de archivo ha procurado preservar y reconstruir las condiciones originales y situadas de la producción documental, a pesar del paso del tiempo y de los traslados de espacio.

Sin embargo, el tratamiento archivístico comenzó a sufrir grandes cambios y derivaciones ante la asimilación y penetración de las tecnologías digitales en los procesos de gestión de las instituciones públicas y privadas (Voutssas, 2011). Las propiedades de los documentos de archivo hoy están siendo profundamente desafiadas por el entorno de internet y la composición y los atributos de los objetos digitales en red, que son inestables y dinámicos (Hui, 2017; Ernst, 2013). Esta problemática nos ha llevado a plantear una pregunta de investigación que, a su vez, exige interrogantes derivados. La pregunta inicial es qué presupuestos teóricos y metodológicos comporta la noción de documento de archivo. Pero además, ¿en qué medida dicha noción sustenta las prácticas de archivo de objetos digitales en internet? ¿Qué similitudes y diferencias se observan entre las propiedades de los documentos de archivo y los objetos digitales en red? ¿Qué desafíos teóricos y metodológicos se abren a futuro?

Este trabajo, de carácter teórico, tiene por objetivo general analizar las premisas teóricas y metodológicas de la noción de documento de archivo, y contrastarlas con las peculiaridades que fueron surgiendo en torno a las prácticas archivísticas de objetos digitales en internet. Como objetivo subsidiario, nos proponemos reflexionar sobre algunos desafíos teóricos y metodológicos que se plantean en el contexto actual, y que parecen introducir discusiones a futuro.

Nuestra hipótesis, que habilita las discusiones sobre esta problemática, es que las propiedades distintivas de los documentos de archivo digitales, a partir del actual entorno de redes, se diferencian ostensiblemente de las propiedades ya estudiadas de los documentos de archivo analógicos. Dicha especificidad exige un tratamiento diferenciado en términos teóricos y metodológicos, como si se estuviera en presencia de paradigmas complementarios. El diseño metodológico definido para esta revisión se basó en un proceso de relevamiento, indagación y cotejo de diversos trabajos y antecedentes teóricos, metodológicos y empíricos, complementado con una instancia de análisis documental para corroborar los supuestos de partida.

Hay antecedentes de estudios dentro del campo que concibieron la irrupción de las tecnologías de la información y la comunicación como elemento decisivo de cambio. Marín Agudelo (2012), por caso, reconoce en la archivística un cambio de paradigma basado en los conceptos que fundamentaron históricamente sus prácticas. Así como distingue a la disciplina 
en su importancia para la salvaguarda del patrimonio documental, ha destacado la necesidad de que las tareas de archivo fueran abordadas en sentido dinámico, lo que exige una modernización de los métodos de trabajo y una ampliación en la accesibilidad de los públicos frente a la omnipresencia digital. Todo a partir de cómo la forma de los documentos, desde la convivencia de soportes, ha supuesto un impacto en las tareas de archivo, en las premisas teóricas y metodológicas heredadas y, por ende, en la cultura documental (92).

El relevamiento de Marín Agudelo reconoce características específicas de los documentos digitales, y desde allí augura un "cambio de mentalidad" para concebir las prácticas a futuro. Ese cambio implica correrse de las tipologías de documentos tangibles para pensar en su utilización; limitar el énfasis en la cuestión del soporte, como premisa heredada, para pensar más en los contextos de producción de los documentos, y atender no sólo a su acceso y conservación, sino a su intervención (2012: 93). La mención, entonces, de un cambio paradigmático dentro de la disciplina hace pie en la noción de documento, y eso involucra aspectos teóricos, de gestión y de tecnologías de información.

Existen, no obstante, perspectivas teóricas que han desbordado desde distintos campos de estudio ${ }^{2}$ las condiciones para definir el objeto archivístico y las entidades documentales. Trabajos como los de Derrida (1997), Farge (1991) y Groys (2016), o tesis como la de Tello (2018) en Latinoamérica, dan cuenta en las últimas décadas de posiciones que advierten sobre el carácter inorgánico de la noción de archivo, contrario a la constitución de la doctrina moderna de la disciplina, ${ }^{3}$ a partir del interrogante sobre qué puede considerarse (y qué tiene dentro) un registro documental y cómo se definen las condiciones para que exista archivo más allá de las premisas historiográficas, es decir, del ordenamiento y la restauración de determinados sucesos "tal como sucedieron” (Tello, 2018: 47). La reflexión sobre ese carácter inorgánico, que puede pensarse en términos sociodiscursivos y culturales tanto que institucionales, cuestiona la afirmación del archivo en tanto entidad dada, regida por sus propias normas, que sólo debe ser guiada hacia un estado original, y también la naturaleza de un documento archivable. No profundizaremos en estas líneas pero vale la mención porque estos abordajes dan cuenta de una

2 Filosóficos, políticos e incluso artísticos, en general vinculados al pensamiento posestructuralista.

3 Nos referimos a textos fundacionales. En el Handleiding voor het Ordenen en Beschrijven van Archieven, escrito por Muller, Feith y Fruin (1910) y llamado "Manual holandés", se expone como propósito de la ciencia de los archivos la identificación del orden original de los documentos. Por su parte, el Principio de respeto al orden original de los documentos fue enunciado por von Seybel en 1881 y difundido por el "Manual holandés" en 1898. 
diversificación conceptual previa a la digitalización que, incluso, ha introducido desafíos en torno a la naturaleza de los archivos digitales.

La siguiente sección presenta los presupuestos teóricos y metodológicos del concepto de documento de archivo dentro de lo que llamamos el paradigma archivístico analógico. A continuación caracterizamos la irrupción de las tecnologías de la información digital dentro de ese paradigma; luego analizamos las características que implica la práctica de archivo de objetos digitales en internet y su relación con los presupuestos teóricos y metodológicos desarrollados, además de señalar similitudes y diferencias entre las propiedades de los documentos de archivo y los objetos digitales en red. Por último, reflexionamos acerca de desafíos teóricos y metodológicos que se abren en torno a la noción de documento de archivo en el contexto actual, proponiendo algunas líneas para nuevas aproximaciones.

\section{El PARADIGMA ARCHIVÍSTICO ANALÓGICO}

El archivo, según las premisas dominantes de la disciplina, no responde a un interés de colección o de almacenamiento circunstancial. Su constitución implica la reunión, organización y preservación de documentos, de la más diversa índole, enlazados por su origen (Cortés Alonso, 1981). Por un lado, esto lo diferencia de otras unidades profesionales de información, como las bibliotecas, que crean colecciones con el propósito de responder a las demandas informativas de los usuarios (Lee, 2000). Por otro lado, también lo distingue de simples cúmulos de documentos, ya que su existencia supone una agrupación documental organizada a partir de principios teóricos y procesos metodológicos específicos.

De acuerdo con el Diccionario de Terminología Archivística, el archivo consiste en el "conjunto orgánico de documentos" producidos o recibidos por personas físicas o jurídicas, públicas o privadas, en el ejercicio de sus funciones (Subdirección General de los Archivos Estatales, 1995). De igual manera como se desagregan las muñecas rusas, los archivos constituyen conjuntos complejos, compuestos de unidades de menor tamaño que, en último término, pueden alcanzar a reunir miles de documentos simples.

Atendiendo a la escala de lo general a lo particular, podemos distinguir tres unidades documentales fundamentales (Tanodi, 2015). En primer lugar, la unidad máxima y más completa es el fondo. Ésta representa la totalidad de la documentación producida o recibida por un sujeto productor -individual o colectivo- en el desarrollo de sus funciones (Heredia Herrera, 1991). El fondo puede dividirse en secciones y subsecciones, teniendo en cuenta la 
estructura organizacional del organismo o sus competencias. En segundo lugar, la unidad documental intermedia es la serie, que se compone del conjunto de documentos obtenidos por el desarrollo de una misma actividad y regulados por la misma norma de procedimiento (Subdirección General de los Archivos Estatales, 1995). En tercer lugar, la unidad menor o pieza documental es el documento simple, de carácter indivisible (Cortés Alonso, 1981). Éste consiste en "toda expresión en lenguaje natural o convencional y cualquier otra expresión gráfica, sonora o en imagen, recogidas en cualquier tipo de soporte material, incluso los soportes informáticos" (Subdirección General de los Archivos Estatales, 1995).

Sin embargo, el documento de archivo tiene ciertas propiedades que lo distinguen de cualquier registro de información en un soporte material. Éstas radican, siguiendo a Schellenberg (1965), en su carácter seriado, génesis, exclusividad e interrelación. No se trata de documentos sueltos: los documentos de archivo surgen uno a uno en el desarrollo de las actividades del organismo productor, por lo que pueden identificarse series. Dado que su origen está estrechamente ligado con el sujeto -individual o colectivo- que los produce, son resultado y reflejo de las funciones que éste desempeña. Además, la información que contiene cada documento de archivo es exclusiva, porque generalmente no se expresa con idéntica extensión e intensidad en otros documentos. Por último, las piezas documentales no tienen sentido aisladamente, sino sólo por su pertenencia a agrupaciones documentales mayores y las relaciones establecidas entre sí (Schellenberg, 1965).

Para Cortés Alonso (1981), los documentos de archivo presentan tres particularidades. En primer lugar, su unicidad. A diferencia de los documentos bibliográficos, la producción de documentos de archivo no es múltiple. Los documentos originales son únicos y su eliminación representa la pérdida total. En segundo lugar, están situados espacial y temporalmente. Dado que se obtienen como resultado de una actividad pública o privada, ésta los circunscribe y los hace semejantes a los que de ella resultan. En tercer lugar, no se tratan de modo aislado sino formando series de unidades documentales originadas por la misma actividad.

A diferencia de otros tipos de documentos, como los bibliográficos, los documentos de archivo son considerados según este paradigma como testimonios materiales de hechos o actos (Subdirección General de los Archivos Estatales, 1995). De ello se desprende que, en algunos casos, sea posible reconocerles valor administrativo, como testimonio de los procedimientos y las actividades de una entidad administrativa; valor legal, por servir de testimonio ante la ley; valor histórico, por ser fuentes primarias para la historia; valor informativo, por servir de referencia para la reconstrucción de cualquier 
actividad del sujeto productor y también como testimonio de la memoria colectiva, entre otros. Dado su valor primario, aquél que va unido a la finalidad inmediata por la cual el documento ha sido producido, y su valor secundario, que depende de la utilidad que se pueda deducir al concluir su gestión administrativa, la metodología de trabajo archivístico se ocupa de resguardar sus propiedades distintivas (Cruz Mundet, 1996).

El tratamiento archivístico de los documentos supone la realización de un proceso secuencial integrado por, al menos, las siguientes fases u operaciones consecutivas: identificación, clasificación, ordenación, instalación, descripción, valoración, selección, difusión (Heredia Herrera, 1991; Otárola Sáenz, 2018; Tanodi, 2015). En primer lugar, busca restablecer el esquema organizacional o funcional que produjo el conjunto documental, distinguiendo grupos o secciones, subsecciones y series documentales. Una vez que fue reconstruido, se ordenan internamente las unidades documentales que integran dichos conjuntos. A continuación, los documentos son instalados en los depósitos, teniendo en cuenta su soporte material y las condiciones especiales requeridas para su conservación. Posteriormente, se procede a la descripción archivística, para identificar y describir el contexto y contenido de los documentos de archivo, con el fin de hacerlos accesibles a los usuarios. La fase de valoración y selección implica un proceso de análisis de los valores primarios y secundarios de los documentos y evaluación de las series documentales para determinar sus plazos de conservación. Por último, la difusión busca promover la utilización de los fondos documentales del archivo, a través de exposiciones, conferencias y eventos culturales, entre otros.

En definitiva, el servicio archivístico no se restringe a procesar los documentos para ofrecerlos al público que los solicita, sino que implica, primordialmente, un reconocimiento y un ejercicio de autoridad: la facultad de decretar su valor archivístico, que justifica su conservación permanente (Cortés Alonso, 1981; Subdirección General de los Archivos Estatales, 1995). Cierto es que existen otras operaciones que forman parte del proceso archivístico, como la restauración de documentos; sin embargo, las fases que mencionamos son las etapas mínimas del tratamiento documental, que no podrían ser alteradas en el orden ni suprimidas en su ejecución, de acuerdo con el método de archivo (Cortés Alonso, 1981; Tanodi, 2015).

Los pilares conceptuales fundamentales sobre los que se sustenta esta metodología corresponden a los principios que emanan de la teoría francesa Respect des fonds y la alemana de Registraturprinzip. Por un lado, el Principio de procedencia, cuya formulación proviene de Natalis de Wailly en 1841, establece que los documentos producidos por una persona física o jurídica no deben mezclarse con los de otras entidades productoras de documentos (Du- 
chein, 1977). Por otro lado, el Principio de respeto al orden original de los documentos, enunciado por Henrich von Seybel en 1881 y difundido por el manual de Muller, Feith y Fruin en 1898, dispone que los documentos de cada fondo deben mantenerse en el orden dado por la oficina de origen, en lugar de ser ordenados por asuntos, temas o materias (Cruz Mundet, 2011). Desde fines del siglo XIX, estos postulados han orientado el desarrollo teórico, la praxis y las políticas archivísticas, a tal punto que llegaron a reconocerse como un paradigma de la comunidad disciplinaria internacional (Cruz Mundet, 2011). Los centenarios principios de procedencia y de respeto al orden original de los documentos han aportado coherencia y solidez a este campo de conocimiento científico y de saber profesional.

Asimismo, este paradigma ha sido complementado con la teoría del ciclo de vida de los documentos (Records Lifecycle), propuesta por Philip Coolidge Brooks en 1940 (Cruz Mundet, 2011). Esta formulación teórica establece relaciones analógicas entre los documentos y los organismos biológicos -la condición orgánica, y cuestionada desde otras disciplinas-, sosteniendo que el documento de archivo nace -fase de creación-, vive -fase de mantenimiento y uso-, y muere -fase de eliminación-. Esto ha dado justificación a la gestión de los documentos (Records management) desde la creación de dichas unidades, dado que permite contar con ellas para la toma de decisiones en el desarrollo de actividades de la entidad productora (Cruz Mundet, 2011).

Recapitulando, presentamos en la Tabla 1 una síntesis de los fundamentos teóricos y metodológicos en torno a la noción de documento de archivo en el paradigma archivístico dominante, construido a partir de procesos de producción documental soportados analógicamente.

\begin{tabular}{|l|l|l|}
\hline $\begin{array}{l}\text { Documento de archivo en } \\
\text { el paradigma analógico }\end{array}$ & \multicolumn{1}{|c|}{ Premisas teóricas } & \multicolumn{1}{|c|}{ Premisas metodológicas } \\
\hline $\begin{array}{l}\text { Propiedades del documento } \\
\text { de archivo }\end{array}$ & $\begin{array}{l}\text { Carácter seriado } \\
\text { Génesis } \\
\text { Exclusividad } \\
\text { Interrelación } \\
\text { Unicidad } \\
\text { Carácter situado }\end{array}$ & $\begin{array}{l}\text { El método de archivo debe } \\
\text { resguardar las propiedades } \\
\text { distintivas de los documentos. } \\
\text { Esto habilita al reconocimiento de } \\
\text { su valor archivístico. }\end{array}$ \\
\hline $\begin{array}{l}\text { Tratamiento archivístico de } \\
\text { los documentos }\end{array}$ & $\begin{array}{l}\text { Proceso secuencial, formado por } \\
\text { operaciones consecutivas. }\end{array}$ & $\begin{array}{l}\text { Las operaciones mínimas no } \\
\text { pueden ser alteradas en orden ni } \\
\text { suprimidas en su ejecución. }\end{array}$ \\
\hline
\end{tabular}

Tabla 1. Presupuestos en torno al documento de archivo Fuente: elaboración propia a partir de Cortés Alonso (1981), Cruz Mundet (1996), Schellenberg (1965) y Tanodi (2015) 


\section{La irrupción de las tecnologías de la información digital y la emergencia del documento electrónico}

A partir de la década de 1980, la expansión de las tecnologías digitales en diversos ámbitos productivos habilitó el surgimiento de nuevas operaciones archivísticas, así como también la modificación de las ya existentes. Por ejemplo, a partir de la práctica sociotécnica de captar y almacenar información en formato imagen emergió la digitalización documental (Mena Múgica y González Crespo, 2013). Si bien el procedimiento ya existía desde tiempos remotos (Gille, 1953), el rasgo novedoso de la operación radicaba en la conversión de información existente en soportes analógicos, como el papel, las cintas de cassette, las películas fotográficas o los discos de vinilo, en flujos de bits.

Sin embargo, la incorporación de nuevas operaciones sociotécnicas en las prácticas de archivo no implicó una transformación de los fundamentos teóricos y metodológicos de la disciplina. Siguiendo el ejemplo de la digitalización, este procedimiento se orientó a garantizar la conservación preventiva de los documentos originales, evitando su manipulación durante las consultas, y brindar una mayor rapidez y precisión en la recuperación de información, facilitando a los usuarios el acceso simultáneo y remoto a los documentos (Mena Múgica y González Crespo, 2013; SNDH, 2019).

En el orden teórico, algunas discusiones conceptuales se desarrollaron a partir de los cambios observados en la producción documental en soporte digital desde la fase de creación de los documentos (Álvarez Rodríguez y Rodríguez, 2005; García-Morales, 2013; Térmens Graells, 2014; Voutssas, 2011). A diferencia de los documentos analógicos digitalizados, los documentos digitales -también llamados electrónicos (Álvarez Rodríguez y Rodríguez, 2005; Martín-Pozuelo Campillos, 2003) - nacen en un soporte numérico que les confiere determinadas características específicas. Según Kallinikos, Aaltonen y Marton (2010: 10): "[...] digital documents have a double mode of existence, being composed by the content arrangement they mediate plus the operations by which this content is assembled and maintained". Por lo tanto, la práctica de archivo de este tipo de documentos exige atender a los atributos y la textura compositiva de su funcionamiento.

En relación con el tratamiento documental, el concepto de la continuidad de los documentos (Records continuum) propuesto por Frank Upward (1996; 1997) a mediados de la década de 1990 buscó superar la separación taxativa entre las etapas del mencionado ciclo vital. En la búsqueda de un marco superador de la concepción predominante, esta perspectiva -influenciada por el pensamiento posmoderno- sugirió el abandono del control secuencial en 
la gestión documental, postulando que los sistemas deben en cambio adoptar un enfoque de continuidad (Cruz Mundet, 2011).

Por su parte, el Foro DLM (Données Lisibles par Machine) de la Comisión Europea definió que los documentos electrónicos de archivo (electronic record) cuentan con los siguientes elementos: contenido, estructura, contexto y presentación (DLM-Forum, 1997). Dado que el registro de información es independiente a su presentación o output, se determinó que los elementos a archivar son los tres primeros (DLM-Forum, 1997).

En cuanto al contenido, éste puede incluir diferentes tipos de datos: texto, números, tablas, imágenes, gráficos, sonido y video, y enlaces de hipertexto. Respecto del segundo elemento, la estructura lógica del documento puede ser muy diferente de la estructura física del registro, y puede estar incorporada en él, en la base de datos, o hallarse separadamente. Por último, el contexto debe describirse en un documento asociado, incluyendo los metadatos técnicos -entorno de hardware y software, incluidos los números de versión, estructura de archivos, una descripción de los datos y un historial de enlaces con otros registros- y una descripción del contexto administrativo involucrado (DLM-Forum, 1997).

A su vez, en el ámbito regional europeo, las discusiones conceptuales del Foro DLM desembocaron en la creación del Model Requirements for the Management of Electronic Documents and Records (MoReq), un instrumento normalizador que fija los requerimientos básicos para programas de gestión de documentos electrónicos de archivo (Gómez, 2010). Se trata de un modelo de requisitos para el desarrollo de Sistemas de Gestión Electrónica de Documentos de Archivo (SGDEA), publicado en 2001 y reeditado en 2008 y 2010 (MoReq ${ }^{\circledR}, 2020$. https://sysresearch.org/moreq/).

En síntesis, las definiciones conceptuales que surgieron sobre documentos electrónicos y su tratamiento archivístico no han salido de la órbita de lo que abordamos como documento de archivo desde el paradigma tradicional. En todo caso, los nuevos términos e instrumentos buscaron extender y adecuar los fundamentos teóricos y metodológicos existentes a la creciente producción documental en soporte digital. La Tabla 2 presenta una síntesis de las premisas y definiciones hasta aquí relevadas. 


\begin{tabular}{|l|l|l|}
\hline \multicolumn{1}{|c|}{$\begin{array}{c}\text { Documento electrónico de } \\
\text { archivo }\end{array}$} & \multicolumn{1}{|c|}{ Premisas teóricas } & \multicolumn{1}{c|}{ Premisas metodológicas } \\
\hline $\begin{array}{l}\text { Propiedades del documento elec- } \\
\text { trónico de archivo }\end{array}$ & $\begin{array}{l}\text { Los elementos que los } \\
\text { constituyen son contenido, } \\
\text { estructura, contexto y pre- } \\
\text { sentación. }\end{array}$ & $\begin{array}{l}\text { Los elementos que deben ser pre- } \\
\text { servados son los tres primeros. }\end{array}$ \\
\hline $\begin{array}{l}\text { Tratamiento archivístico de los } \\
\text { documentos electrónicos }\end{array}$ & $\begin{array}{l}\text { Proceso continuo, aten- } \\
\text { diendo a la transaccionali- } \\
\text { dad y la contextualidad. }\end{array}$ & $\begin{array}{l}\text { Los sistemas de gestión de } \\
\text { documentos electrónicos deben } \\
\text { cumplir las especificaciones } \\
\text { establecidas en MoReq. }\end{array}$ \\
\hline
\end{tabular}

Tabla 2. Presupuestos en torno al documento de archivo Fuente: elaboración propia a partir de DLM-Forum (1997) y Upward $(1996,1997)$

\section{EL ARCHIVO DE OBJETOS DIGITALES EN INTERNET}

Los procesos de archivo desarrollados en internet durante las últimas dos décadas plantean algunos cambios radicales frente a las prácticas sociotécnicas del paradigma dominante (Kallinikos, Aaltonen y Marton, 2010; Ernst, 2013). Tal y como lo habían anticipado las directrices del DLMForum (1997), "The context described in the documentation may be extremely complex if the record is integrated in a network architecture" (13). En este sentido, las prácticas de archivo de objetos digitales en red se alejan de los fundamentos teóricos y metodológicos sostenidos en la disciplina y formulados inicialmente para documentos de naturaleza analógica. Esta alteración significativa repercute especialmente en la noción de documento de archivo y en la consideración de las prácticas archivísticas en el contexto de internet. De allí nace la pregunta sobre cuáles son sus características específicas, sus similitudes y diferencias con la noción original, y los desafíos teóricos y metodológicos que se abren teniendo en cuenta que el entorno de redes es cada vez más omnipresente en diversas actividades humanas, sobre todo de orden social y cultural.

Para considerar las particularidades del archivo de objetos digitales en internet recuperamos una investigación empírica realizada por Kallinikos, Aaltonen y Marton (2010) acerca del proyecto Internet Archive, gestionado por una organización sin fines de lucro y dedicado a la preservación de objetos digitales de la Web.

Internet Archive nació en 1996 a partir de la iniciativa del ingeniero informático Brewster Kahle. Se aloja en el dominio archive.org y fue pensado, inicialmente, como una iniciativa para que todo objeto digital dedicado a la producción cultural -sitios web, películas, libros, obras musicales, incluso 
software- que navegara por la Web no llegara a desaparecer sin dejar rastros. El método para alcanzar ese objetivo se basa en la generación de copias de respaldo - a la manera de capturas, como una fotografía- de cada documento publicado, a partir de su Localizador Uniforme de Recursos (URL, según su sigla en inglés). Esto lo realiza un motor de búsqueda creado por Kahle, denominado Alexa, que copia cada objeto digital en una base de datos para su indización. De ese modo, Internet Archive contempla una arquitectura de miles de colecciones, cada una correspondiente a un dominio web, que se nutren de capturas con cada modificación de código HTML que van sufriendo las páginas.

Cualquier usuario puede buscar y acceder a los documentos digitales almacenados en Internet Archive a través de una interfaz del motor, WayBack Machine, que se ofrece públicamente para la consulta. Así, mediante este buscador, los usuarios desde cualquier parte del mundo pueden recuperar documentos online que, por distintas vicisitudes, se han vuelto inaccesibles. Estos objetos digitales fueron organizados en la base de datos según cada dominio original y etiquetados con la referencia temporal de su captura.

Teniendo en cuenta, entonces, que dichos objetos se producen y circulan online, el estudio de Kallinikos, Aaltonen y Marton (2010) señala que el proceso de archivo ocurrido en Internet Archive requiere necesariamente contrarrestar algunos de los atributos propios de la condición en red, para obtener objetos digitales que persistan en el tiempo. Concretamente, esto es lo que se busca con la realización de las capturas tomadas a partir de los distintos URL. Lo que permite cada captura es "congelar" el contenido de los documentos en una entidad fija y preservable. Siguiendo a los autores:

Una página web dinámica, por ejemplo, consiste principalmente en instrucciones sobre cómo generar una página web real para que se muestre a un usuario. Procesadas por un servidor web, estas instrucciones recopilan el ensamblaje de varias partes encontradas en varias fuentes. La instantánea tomada no parte de estas instrucciones, sino de la página resultante ensamblada temporalmente y representada como una página HTML para un usuario determinado. (Kallinikos, Aaltonen y Marton, 2010, traducción propia.)

Por lo tanto, el documento archivado no es la página web que se presenta dinámicamente frente al usuario, sino un objeto digital estático, cuya constitución no depende del acceso a las fuentes originales para obtener información actualizada. Asimismo, la temporalidad del documento es establecida en el momento en que se realiza el proceso de captura de la información. Más aún, los objetos digitales en red no presentan una existencia diferenciada previa 
a su archivo: es el proceso mismo de captura el que los torna documentos de archivo, para retomar la noción que abordamos. Es en este sentido que tomamos el trabajo de Kallinikos, Aaltonen y Marton (2010) como antecedente para formular el siguiente interrogante: ¿cuál es el modo de existencia real de los objetos digitales, y cuáles son sus propiedades distintivas?

De acuerdo con Thibodeau (2002), un objeto digital es un objeto de información si su ontología presenta tres condiciones: en primer lugar, ser un objeto físico, una inscripción del código en un medio de almacenamiento dado; en segundo lugar, ser un objeto lógico, como datos reconocidos e interpretados como tales por un software particular; y, en tercer lugar, ser un objeto conceptual, es decir, el output del proceso como se manifiesta en las pantallas, los parlantes, las impresiones, entre otros dispositivos.

Recuperando lo que había planteado el Foro DLM acerca de la prescindencia del archivo de la presentación u output, la condición de objeto conceptual sería entonces desestimada en la práctica de archivo (DLM-Forum, 1997). Desde otra perspectiva, Kirschenbaum (2007) llegó a la misma conclusión al sostener que la tercera condición planteada por Thibodeau (2002) es necesariamente antropocéntrica, porque asume a la percepción humana como la única relevante. A partir de este argumento, Kirschenbaum reflexionó acerca de los objetos digitales desde la primera y la segunda condición, partiendo de los conceptos de materialidad forense y materialidad formal respectivamente (2007: 10).

Por un lado, la materialidad forense es concreta y autográfica. Se basa en el principio de individualización que permite cada inscripción de un código en un dispositivo de almacenamiento dado, a partir de la idea de que no puede haber dos cosas exactamente idénticas en el mundo físico. En términos de la materialidad forense, entonces, podemos ver a un objeto digital dado como su realización física en términos de voltajes, de una distribución de orientaciones magnéticas en una superficie adecuada o de agujeros en un papel o un disco (Blanco y Berti, 2016). Desde esta perspectiva, hay tantos objetos como formas materiales de inscripción del código. Como consecuencia, toda copia "traiciona el principio autográfico" de la materialidad forense (Blanco y Berti, 2016: 202).

Por otro lado, la materialidad formal es abstracta y alográfica. Una misma materialidad formal - una secuencia de bits, por ejemplo- puede tener múltiples materialidades forenses. Desde esta perspectiva, todas las copias gozan de un mismo estatuto. Dicho con otras palabras, constituyen un único objeto (Blanco y Berti, 2016).

Los conceptos presentados por Kirschenbaum (2007) permiten abordar aspectos que no aparecen caracterizados en el modelo tripartito de Thibodeau 
(2002). Además, Kirschenbaum (2007) amplía la posibilidad de múltiples formas de existencia material de los objetos digitales. Sin embargo, su propuesta no advierte un aspecto fundamental: cuando una materialidad formal deja de ser interpretable, pierde su forma de existencia en tanto objeto. Por lo tanto, es el contexto el que determina las condiciones de posibilidad de que los objetos digitales existan como tales.

En este sentido, Hui (2017) hizo un aporte significativo acerca de los medios asociados en los cuales los objetos digitales existen y en función de los que se organizan. Para el autor, un objeto digital tiene sentido sólo dentro de la red que lo incluye, por lo que propone aproximarse a los objetos digitales desde el concepto de relaciones (Hui, 2017: 90-92). Desde esta perspectiva, la identidad misma de los objetos digitales sólo puede considerarse a partir de un marco de referencia -un compilador o intérprete, un sistema operativo, una codificación de imagen o sonido-, por lo que el medio asociado resulta indisociable de su modo de existencia (Blanco y Berti, 2016).

Estas consideraciones sobre la ontología de los objetos digitales revisten importancia en la discusión sobre las prácticas archivísticas en el contexto de internet, que se constituye como un campo de estudios y reflexión cada vez más nutrido. Como dijimos, en el estudio del proyecto Internet Archive, Kallinikos, Aaltonen y Marton (2010) señalaron que el archivo requiere contrarrestar algunos de los atributos propios de la condición en red para la obtención de objetos digitales persistentes. Esas operaciones que alteran los elementos propios de este tipo de objetos permiten observar, en definitiva, que todo objeto digital nacido en el contexto de las redes termina siendo construido durante el proceso de archivo, en lugar de hablar de unidades reunidas, como ocurre con los documentos en las prácticas de archivo convencionales.

Por otra parte, siguiendo la argumentación de Hui (2017), observamos que el contexto situado de estos objetos digitales reside en internet. La red de redes es el medio asociado a partir del cual los objetos digitales se constituyen como tales. Por eso, dado que allí su modo de existencia es dinámico y efímero, su archivo supone la separación de la materialidad formal del medio asociado, para la constitución de entidades estables y preservables a través del tiempo. Esto se ubica en las antípodas de los presupuestos teóricos y metodológicos del paradigma archivístico analógico, dado que el contexto original y situado de producción debe ser aislado para constituir las unidades documentales y garantizar su perdurabilidad. La característica distintiva del archivo de los objetos digitales en red, frente a los documentos de archivo analógicos, no radica solamente en su diferente inscripción en el soporte material, sino especialmente en la abstracción de su condición en red y la transformación a un modo de existencia fijo y accesible más allá del entorno de internet (Kallinikos, Aaltonen y Marton, 2010; Ernst, 2013). 
En relación con las propiedades de los objetos digitales, Kallinikos, Aaltonen y Marton (2010) proponen cuatro aspectos para dar cuenta de su naturaleza. Primero, el alto grado de edición que admiten -por ejemplo, páginas wiki. Segundo, el grado de interactividad -ostensible en foros de discusión y redes sociales. Tercero, el grado de apertura -la capacidad de ser rastreados por motores de búsqueda-y, cuarto, el altísimo grado de distribución -páginas web dinámicas, por ejemplo, que toman contenidos de distintas bases de datos, así como las colecciones de imágenes. El contenido online se caracteriza por ser altamente transfigurable y no se presenta, como afirma Hjørland (2000), en forma de documentos bien definidos, fácilmente identificables, con límites claros o evidentes.

Las propiedades de los objetos digitales en red y las operaciones necesarias para su archivo implican una transmutación de implicancias teóricas, metodológicas y ontológicas respecto del tratamiento documental sistematizado por el paradigma analógico. En el ejemplo analizado, el archivo no recoge entidades ya delimitadas, sino que busca construir los límites que demarcan y hacen un documento archivable (Kallinikos, Aaltonen y Marton, 2010). Desde 1996, Internet Archive produce objetos digitales persistentes que se sustentan en procedimientos completamente diferentes de los seguidos por las instituciones archivísticas. Es posible advertir que los principios teóricos de respeto a la procedencia y el orden original de los documentos no rigen, ni pueden regir, el tratamiento de los objetos digitales en red, sino que estos son creados de manera tal de transformar los flujos de bits transfigurables en un documento de archivo identificable sin el grado de capacidad de edición, interactividad, apertura y distribución que el original ofrece (Kallinikos, Aaltonen y Marton, 2010).

Vale señalar, no obstante, que la distancia observada entre métodos de reunión o de construcción del documento archivable no surgió con el soporte digital y la irrupción de internet. Desde las premisas del paradigma archivístico analógico podría sugerirse que las propiedades de los objetos digitales en red dan cuenta de una naturaleza anarchivística, siguiendo el concepto de Derrida (1997): una resistencia de origen a la condición archivable, que parece estar más cerca de propiciar un "borramiento de huellas" (22) que una estabilización para la preservación documental. Tello (2018) también concibe dicha condición al margen de la discusión ontológica sobre los documentos, porque implica al trabajo de quien interviene los grupos documentales buscando construir -y discutir- un método. El anarchivismo, para Tello (2018), puede pensarse como un movimiento que altera los sistemas normalizados de registros (56). 
Desde posiciones ajenas a la archivística, la afirmación de Kallinikos, Aaltonen y Marton (2010) respecto a la naturaleza del objeto digital en tanto objeto construido, cuyo valor se realiza a partir del aislamiento de sus condiciones originales de producción y circulación, recupera los complejos intentos de definir qué es un documento archivable y cuándo y de qué modo se despliega su condición -y la condición del archivo-. Si un objeto digital en red se construye necesariamente a partir de las propiedades de su entorno, y se convoca -es ensamblado- cada vez que un usuario lo demanda, su raíz ontológica no puede desligarse de la noción de intervención, eso que Derrida (1997) afirmó, en los albores de internet, bajo la idea de que la archivación produce tanto como registra el acontecimiento.

Puede pensarse que las redes refuerzan el cuestionamiento disciplinar sobre la entidad documental y la consecuente naturaleza orgánica del archivo, pero también puede advertirse la posibilidad de estar frente a asuntos paralelos. El archivo de objetos digitales en red requiere de una intervención sobre el contexto de la materialidad formal para constituir al objeto en cuanto tal y preservarlo en el tiempo, y este argumento es consistente para discutir si el modo de existencia de los objetos digitales en red y sus propiedades distintivas no presentan una especificidad tal que exija tratarlos bajo fundamentos teóricos y metodológicos diferentes de los que rigen para los documentos de archivo analógicos. La Tabla 3 resume la discusión de este apartado.

\begin{tabular}{|l|l|l|}
\hline \multicolumn{1}{|c|}{ Objetos digitales en internet } & \multicolumn{1}{|c|}{ Aspectos teóricos } & \multicolumn{1}{c|}{ Aspectos metodológicos } \\
\hline $\begin{array}{l}\text { Propiedades de los objetos } \\
\text { digitales en red }\end{array}$ & $\begin{array}{l}\text { Entidades dinámicas y efímeras. } \\
\text { Capacidad de edición, interactivi- } \\
\text { dad, apertura y distribución. }\end{array}$ & $\begin{array}{l}\text { Se requiere abstraer su condición } \\
\text { en red para obtener entidades } \\
\text { fijas y preservables en el tiempo. }\end{array}$ \\
\hline $\begin{array}{l}\text { Tratamiento documental de los } \\
\text { objetos digitales en red }\end{array}$ & $\begin{array}{l}\text { Proceso de constitución de } \\
\text { objetos digitales estables, cuya } \\
\text { temporalidad se establece en el } \\
\text { momento de archivo. }\end{array}$ & $\begin{array}{l}\text { Se requiere aislar la materiali- } \\
\text { dad formal del medio asociado } \\
\text { (internet). }\end{array}$ \\
\hline
\end{tabular}

Tabla 3. Aspectos teóricos y metodológicos del archivo de objetos digitales en la red Fuente: elaboración propia a partir de Kallinikos, Aaltonen y Marton (2010); Kirschenbaum (2007); Hui (2017)

\section{Consideraciones FinAles}

Hemos relevado un conjunto de premisas teóricas y metodológicas centrales para analizar y actualizar la noción de documento de archivo. Partimos de los presupuestos fundantes del paradigma archivístico analógico para contrastarlos con la expansión del soporte digital y la emergencia de documentos 
electrónicos, lo que introdujo discusiones más complejas en torno a las propiedades de los documentos y la ontología de los objetos digitales en red.

En el contraste de las premisas fundantes con las prácticas de archivo de objetos digitales se pueden observar, en principio, algunas similitudes. Como afirmó Hui (2017), es el contexto -relacional- el que determina las condiciones de posibilidad para que los objetos digitales se constituyan como tales. Esto coincide con la noción primigenia de documentos de archivo: no se trata sólo de documentos que portan información, como los bibliográficos, sino que presentan la particularidad de estar enlazados por su origen.

Sin embargo, como planteamos en la hipótesis inicial, también es posible advertir diferencias profundas entre los presupuestos teóricos y metodológicos de los respectivos procesos, siempre dentro del campo de estudio de la archivística. Mientras que el paradigma archivístico analógico propone la reunión de documentos preexistentes, el archivo de objetos digitales en red los construye. Para ello, este último separa la materialidad formal del medio asociado -internet-, contrarrestando así los atributos de su condición en red. En cambio, el contexto original de producción es el que la teoría archivística insiste en preservar, más allá de otras perspectivas teóricas que lo discuten, dado que la naturaleza de los documentos de archivo se sustenta en los lazos de su origen. Las piezas documentales no tienen sentido aisladamente, sino en su interrelación.

Cabe señalar además que, mientras los documentos de archivo están situados espacial y temporalmente, la temporalidad de los objetos digitales en red es otro de los puntos críticos de análisis ya que se establece en el momento de su constitución y archivo. Desde el punto de vista del carácter situado, el paradigma archivístico analógico se ha preocupado por resguardar el valor archivístico de los documentos para servir de testimonio material de los hechos o actos, lo que busca seguir sosteniendo la noción de archivo orgánico. En cambio, el valor archivístico de los objetos digitales en red, que permite su conservación permanente, se construye a partir de la separación de su contexto. Esta intervención va a contramano de la valoración realizada en el paradigma archivístico dominante, y da cuenta de la presencia de propiedades específicas para cada tipo de documento.

Estas constataciones no pretenden conducir a una recuperación de los presupuestos teóricos y metodológicos del paradigma archivístico analógico para "resistir" frente a los procesos -dinámicos, inestables- que actualmente se desarrollan en relación con los objetos digitales en internet. En el otro extremo, tampoco pretenden establecer una mirada crítica hacia la tradición teórica y metodológica que ha guiado sólidamente los procesos de archivo durante cientos de años. En todo caso, la discusión busca abrir el debate sobre 
las grandes transformaciones que están ocurriendo y las implicancias que suponen para el desarrollo de nuevas aproximaciones teóricas y metodológicas.

Es por esto que presentamos, para terminar, algunas problemáticas que signan las discusiones a futuro sobre las prácticas de archivo. En primer término, lo que concierne a la concepción dinámica de todo archivo digital, que ha disparado un cuestionamiento de las operaciones algorítmicas aplicadas a la archivística. Según Ernst (2013), las operaciones primarias del procedimiento archivístico digital no atienden a los contenidos de los documentos, sino a la interconexión logística entre éstos. La web, de hecho, no se define por sus contenidos sino por la conjunción de aplicaciones de softwares y sus protocolos de transmisión. Es así que toda práctica de archivo en internet termina adecuándose a una lógica poshumana que muestra cómo la evolución de las capacidades de procesamiento excede a las intervenciones de cualquier archivista. El punto crítico es ubicar conceptualmente a esta nueva realidad en la que el archivo de objetos digitales comienza a ser establecido por procesos algorítmicos, en vez de ser comandado por especialistas. Ernst (2013) habla, por lo anterior, de todo proceso de archivo digital como una metáfora de archivo, y eso involucra necesariamente a una reconfiguración de las políticas que definen qué debe ser archivado y qué no. Una puja que implica nuevas definiciones tanto a nivel privado, público y estatal.

En segundo lugar, el cambio en las formas de registro y almacenamiento documental exige una adecuación a nuevos tipos de memorias y procesos dinámicos. Esto lleva a profundizar en las nociones de regeneración y (co) producción al pensar las prácticas de archivo a futuro, que también deberán incluir la noción que Pogaçar (2016) denominó microarchivos de la intimidad pública: el paso de la agencia institucional a la agencia individual gracias a las posibilidades que brinda la gestión de medios digitales.

Por último, se destaca un interrogante de Ernst (2013) que conjuga lo anterior. El fuerte de los archivos a futuro, ¿será la tangibilidad o la intangibilidad? ¿El valor archivístico se mantendrá en el reaseguro de los documentos - un patrimonio jurídico o cultural, por ejemplo- o será dirigido a la información en tránsito, tan dinámica como efímera, con el fin del acceso y el uso? Aproximarse a posibles respuestas parece un desafío ineludible en el marco de la disciplina. 
Agradecemos especialmente el apoyo recibido por parte del proyecto de investigación Producción, almacenamiento y circulación del conocimiento artístico e intelectual. Indagación multidisciplinar sobre archivos, repositorios y formatos de publicación, dirigido por el Dr. Marcelo Casarín y radicado en el Centro de Estudios Avanzados de la Facultad de Ciencias Sociales de la Universidad Nacional de Córdoba (Res. SeCyT-UNC N. . 411/2018).

\section{REFERENCIAS}

Alberch i Fugueras, Ramón y José Ramón Cruz Mundet. 1999. ¡Archivese! Los documentos del poder. El poder de los documentos. Madrid: Alianza Editorial.

Álvarez Rodríguez, Mariela y José David Rodríguez. 2005. "El documento electrónico: ¿qué características debe cumplir de cara a las organizaciones del siglo XXI?". Revista Códice (1).

Blanco, Javier y Agustín Berti. 2016. "No hay hardware sin software: Crítica del dualismo digital”. Quadranti. Rivista internazionale difilosofia contemporanea 4 (1-2): 197-214. https://ri.conicet.gov.ar/handle/11336/96809

Cortés Alonso, Vicenta. 1981. "Los documentos y su tratamiento archivístico". B. Anabad 31 (3): 365-381.

Cruz Mundet, José Ramón. 1996. Manual de archivística. 2a. ed. Madrid: Pirámide.

Cruz Mundet, José Ramón. 2011. "Principios, términos y conceptos fundamentales", en Administración de documentos y archivos. Textos fundamentales, dirigido por José Ramón Cruz Mundet, 15-35. Madrid: Coordinadora de Asociaciones de Archiveros.

Derrida, Jacques. 1997. Mal de archivo. Una impresión freudiana. Madrid: Trotta.

DLM-Forum. 1997. Guidelines on best practices for using electronic information. How to deal with machine-readable data and electronic documents. Luxembourg: Office for Official Publications of the European Communities. https://www.culturaydeporte.gob.es/dam/jcr:5239db3c-d76e-459c-be4b8df60150bc91/gdlines-dlm-forum.pdf

Duchein, Michel. 1977. "Le "respect des fonds " en archivistique: principes théoriques et problèmes pratiques". Gazette des archives (97): 71-96. https://www.persee.fr/doc/gazar_0016-5522_1977_num_97_1_2554

Ernst, Wolfgang. 2013. Digital memory and the Archive. Minneapolis: University of Minnesotta Press.

Farge, Arlette. 1991. La atracción del archivo. Valencia: Edicions Alfons el Magnánim.

García-Morales, Elisa. 2013. Gestión de documentos en la e-administración. Barcelona: Editorial de la Universitat Oberta de Catalunya.

Gille, Bertrand. 1953. "Equisse d'un plan de normalisation pour le microfilmage des archives". Archivum (3): 87-104.

Gómez, Ruth. 2010. "MoReq: Modelo europeo de requerimientos para un sistema de gestión de documentos electrónicos de archivo". Anuario Escuela de Archivología (2): 71-73.

https://revistas.unc.edu.ar/index.php/anuario/article/view/4208 
Groys, Boris. 2016. Arte en flujo. Ensayos sobre la evanescencia del presente. Buenos Aires: Caja Negra.

Heredia Herrera, Antonia. 1991. Archivística general. Teoría y práctica. 5a. ed. Sevilla: Diputación Provincial de Sevilla.

Hui, Yuk. 2017. “¿Qué es un objeto digital?”. Virtualis 7 (15): 81-96.

Hjørland, Birger. 2000. "Documents, memory institutions and information science". Journal of Documentation 56 (1): 27-41. https://doi.org/10.1108/EUM0000000007107

Kallinikos, Jannis, Aleksi Aaltonen y Attila Marton. 2010. "A theory of digital objects". First Monday 15 (6). https://doi.org/10.5210/fm.v15i6.3033

Kirschenbaum, Matthew G. 2007. “Introduction: 'Awareness of the Mechanism”, en Mechanisms: New Media and the Forensic Imagination, 1-23. Cambridge: The MIT Press.

Lee, Hur-Li. 2000. "What is a collection?". Journal of the American Society for Information Science 51 (12): 1106-1113.

Marín Agudelo, Sebastián Alejandro. 2012. "Estado de la archivística en América Latina 2000-2009. Perspectivas teóricas y aproximaciones conceptuales”. Investigación Bibliotecológica 26 (57): 77-101. https://doi.org/10.22201/iibi.0187358xp.2012.57.33840

Martín-Pozuelo Campillos, María Paz. 2003. "Los documentos electrónicos: oportunidades para el profesional de la archivística”. Trabajo presentado en la Jornada Técnica La administración electrónica y los archivos: amenazas y oportunidades para la archivística, Toledo, España, 10 de julio.

Mena Múgica, Mayra M. y Arien González Crespo. 2013. "Una imagen, mil palabras: la digitalización como estrategia de preservación de documentos archivísticos", en Archivos electrónicos. Textos y contextos II, coordinado por Alicia Barnard Amozorrutia, 9-36. Puebla: Benemérita Universidad Autónoma de Puebla.

Otárola Sáenz, Mellany. 2018. Descripción de documentos. San José: Ministerio de Cultura y Juventud, Dirección General del Archivo Nacional.

Pogaçar, Martin. 2016. Media archaeologies, micro-archives and stoytelling. Re-presencing de past. London: Palgrave Macmillan.

Schellenberg, Theodore Roosevelt. 1965. The Management of Archives. New York: Columbia University Press.

SNDH (Sistema Nacional de Documentación Histórica). 2019. Guía general de digitalización de documentos.

https://www.argentina.gob.ar/sites/default/files/guia_general_de_digitalizacion_de_documentos_vf.pdf

Subdirección General de los Archivos Estatales. 1995. Diccionario de Terminología Archivística. 2a. ed. Madrid: Ministerio de Cultura, Gobierno de España. https://www.culturaydeporte.gob.es/cultura/areas/archivos/mc/dta/portada.html

Tanodi, Aurelio. 2015. Manual de Archivología Hispanoamericana. Córdoba: Brujas.

Tello, Andrés. 2018. "Una archivología (im)posible. Sobre la noción de archivo en el pensamiento filosófico". Síntesis. Revista de filosofía 1 (1): 43-65.

https://doi.org/10.15691/0718-5448Vol1Iss1a234

Térmens Graells, Miquel. 2014. Preservación digital. Barcelona: Editorial de la Universitat Oberta de Catalunya. 
Thibodeau, Kenneth. 2002. "Overview of Technological Approaches to Digital Preservation and Challenges in Coming Years". Trabajo presentando en la Conferencia The State of Digital Preservation: An International Perspective. Washington, Estados Unidos, 24-25 de abril.

https://www.clir.org/pubs/reports/pub107/thibodeau/

Upward, Frank. 1996. "Structuring the records continuum part one. Post-custodial principles and properties". Archives and Manuscripts 24 (2): 268-285.

Upward, Frank. 1997. "Structuring the records continuum part two. Structuration theory and recordkeeping". Archives and Manuscripts 25 (1): 10-35.

Voutssas, Juan. 2011. "Factores culturales, económicos y sociales de la preservación documental digital”. Investigación Bibliotecológica 25 (55): 107-150.

https://doi.org/10.22201/iibi.0187358xp.2011.55.33010

Para citar este texto:

Isoglio, Antonela y Diego Germán Vigna. 2021. "Desafíos teóricos y metodológicos en torno al documento de archivo como objeto digital en la red”. Investigación Bibliotecológica: archivonomía, bibliotecología e información 35 (87): 129-150.

http://dx.doi.org/10.22201/iibi.24488321xe.2021.87.58351 